\title{
Correction to: Proteasome-dependent inactivation of Akt is essential for 12-0-tetradecanoylphorbol 13-acetate-induced apoptosis in vascular smooth muscle cells
}

\author{
Yongna Fan ${ }^{1} \cdot$ Ping Xie ${ }^{1} \cdot$ Hua Zhang ${ }^{1} \cdot$ Shubin Guo ${ }^{2} \cdot$ Dongfeng $\mathrm{Gu}^{3} \cdot$ Mingpeng She $^{1} \cdot$ HuiHua $\mathrm{Li}^{1}$
}

Accepted: 23 November 2021 / Published online: 27 November 2021

(c) Springer Science+Business Media, LLC, part of Springer Nature 2021

\section{Correction to: Apoptosis (2008) 13(12):1401-1409 https://doi.org/10.1007/s10495-008-0272-z}

The original version of this article unfortunately contains errors in figures.

The images for p-AKT, AKT and GAPDH in Fig. 3B, and the image for AKT in Fig. 4B (right panel) were shown incorrectly. The correct Figs. 3B and 4B are given below.

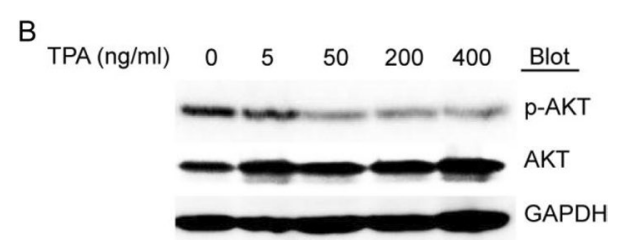

Fig. 3 TPA inhibits Akt phosphorylation. b SMCs were treated with $0,5,50,200$, or $400 \mathrm{ng} / \mathrm{ml}$ TPA for $1 \mathrm{~h}$. The protein levels of Akt were examined using Western blotting with anti-Akt and anti-phospho-Akt antibodies. GAPDH was used as an internal control

The original article can be found online at https://doi.org/10.1007/ s10495-008-0272-z.

HuiHua Li

hhli1995@yahoo.com

1 Department of Pathology and National Laboratory of Medical Molecular Biology, Peking Union Medical College, Institute of Basic Medical Sciences, Chinese Academy of Medical Sciences, Tsinghua University, Beijing 100005, China

2 Department of Acute Medicine, Peking Union Medical Hospital, Beijing, China

3 Division of Population Genetics and Prevention, Cardiovascular Institute and Fuwai Hospital, Chinese Academy of Medical Sciences \& Peking Union Medical College, Beijing 100005, China

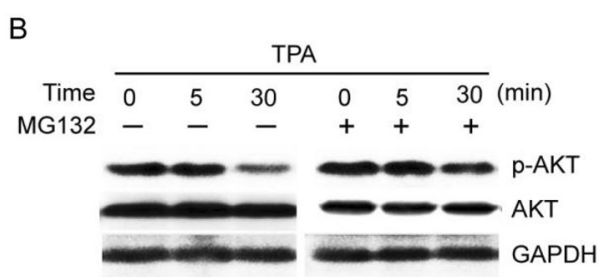

Fig. 4 TPA downregulates the level of Akt phosphorylation through proteasome degradation. b SMCs were treated with vehicle, or MG132 (10 $\mathrm{lM})$ for $30 \mathrm{~min}$, and then incubated with TPA for different time points. Cell lysates were analyzed with Western blotting using anti-Akt and anti-phospho-Akt antibodies. GAPDH was used as an internal control

The corrections do not affect either the validity of the data or the overall conclusions.

Publisher's Note Springer Nature remains neutral with regard to jurisdictional claims in published maps and institutional affiliations. 